\title{
A Novel and efficient magnetic nano-catalyst functionalized with boric acid for the synthesis of symmetric and asymmetric hantzsch esters
}

\author{
Mahsa Sam, Mohammad G. Dekamin* \\ Pharmaceutical and Heterocyclic Compounds Research Laboratory, Department of Chemistry, \\ Iran University of Science and Technology, Tehran, 16846-13114, Iran \\ Tel: +98-21-77240640-50; fax: +98-21-73021584; e-mail: mdekamin@iust.ac.ir
}

\begin{abstract}
An efficient synthesis of polyhydroquinolines (PHQs) and polyhydroacridines (PHAs) via onepot multicomponent reactions of $\beta$-dicarbonyl, different aldehydes, and ammonium acetate catalyzed by a novel nano-catalyst $\mathrm{Fe}_{3} \mathrm{O}_{4} / \mathrm{SiO}_{2}$ functionalized with Boric Acid in EtOH under reflux conditions. It has excellent yield with straightforward workup. The catalyst which been used in this process is a mild heterogeneous catalyst that is environmentally friendly, renewable and recoverable that reused six times without significant loss of its activity.
\end{abstract}

Keywords: polyhydroquinolines (PHQs), polyhydroacridines (PHAs), nano-catalyst, multicomponent reactions, heterogeneous catalyst 


\section{Introduction}

Multicomponent reactions (MCRs) are one-pot reactions.[1] Several descriptive tags are regularly attached to MCRs: they are atom economic, efficient, convergent and exhibit a very high bond-forming-index (BFI); thus, MCRs are usually a good substitute for sequential multistep synthesis.[2] The product of many multi-component reactions is the heterocyclic compounds.[3] Generally known as one of the main groups of nitrogen heterocycles, polyhydroquinolines(PHQs) and polyhydroacridines(PHAs) have become considerably interesting due to various significant pharmacological and therapeutic properties. $[4,5]$ These compounds are representing a significant class of aza-heterocyclic compounds. They enjoy potential biological and pharmacological uses.[6] they are used as anti-malaria, antimicrobial, antifungal, vasodilator, anticancer, bronchodilator, antiatherosclerotic, antitumor, geroprotective, hepatoprotective, antidiabetic activity, anti-cancer, and anti-tumor agents, and as calcium b-blockers, also in the producing of laser color. [7-9]

In an attempt to indicate how applying MNPs would impact the process, this study reports uses of boric acid functionalized MNPs (1) as a novel catalyst in the one pot synthesis of polyhydroquinolines and polyhydroacridines derivatives.

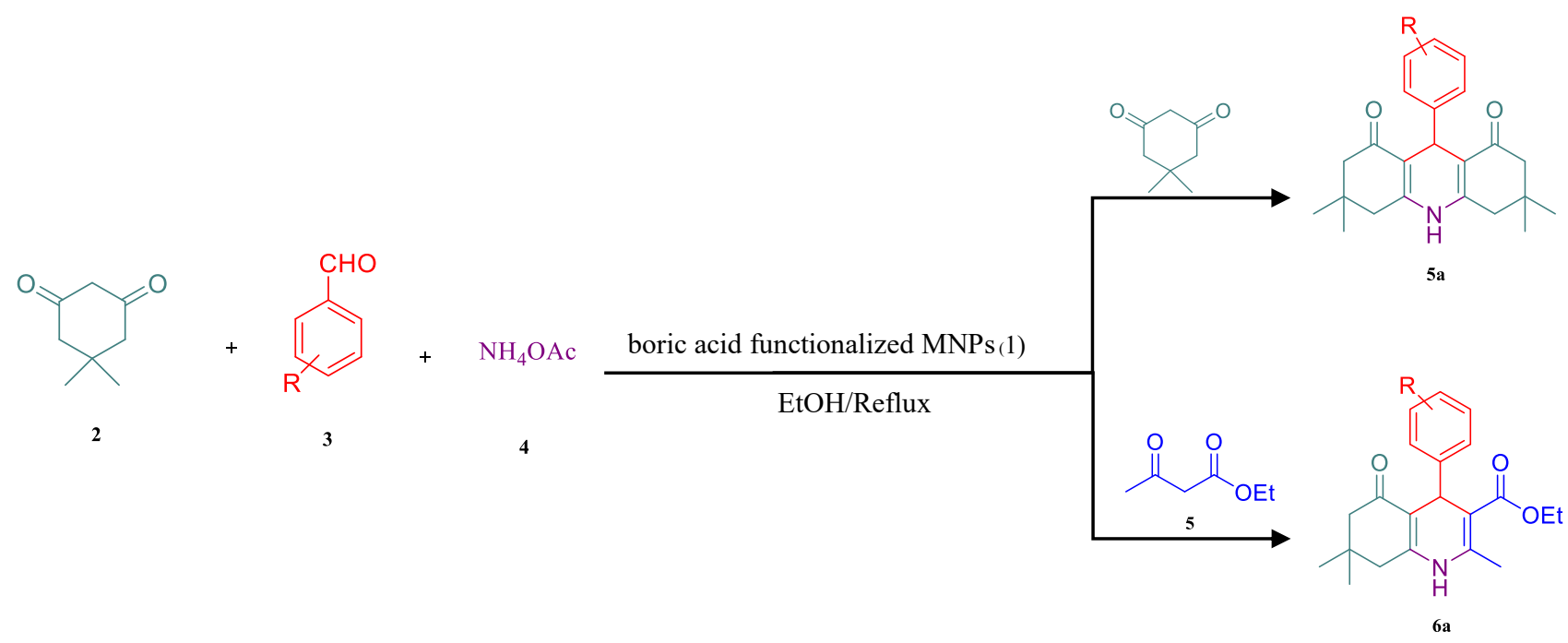

Scheme 1. Synthesis of Polyhydroquinoline and polyhydroacridines derivatives catalyzed by boric acid functionalized MNPs (1) 


\section{Experimental section}

\subsection{General}

Reagents and Apparatus

High-purity chemical reagents were purchased from Merck. All reactions and the purity of the products were monitored by thin-layer chromatography (TLC) using aluminum plates coated with silica gel F254 plates (Merck) using ethyl acetate and n-hexane as eluents. The spots were detected either under UV light or by placing in an iodine chamber. Melting points were determined in open capillaries using an Electrothermal 9100.

General procedure for the synthesis of polyhydroacridines derivatives (5a-b)

In a round-bottomed flask, dimedone $(2,2 \mathrm{mmol})$, varius aldehydes $(3,1 \mathrm{mmol}), \mathrm{NH}_{4} \mathrm{OAc}(4,1$ $\mathrm{mmol})$ and 10mg boric acid functionalized MNPs (1, $10 \mathrm{mg})$ were added to EtOH 96\% (2 mL). The obtained mixture was stirred under reflux condition. After completion of the reaction monitored by TLC (eluent: 25\% v/v EtOAc/n-hexane), EtOH 96\% (3 mL) was added and the catalyst 1 was easily separated from the reaction mixture by an external magnet during recrystallization of the products.

\section{General procedure for the synthesis of Polyhydroquinoline derivatives (6a-d)}

In a round-bottomed flask, dimedone $(2,1 \mathrm{mmol})$, varius aldehydes $(3,1 \mathrm{mmol}), \mathrm{NH}_{4} \mathrm{OAc}(4,1$ $\mathrm{mmol})$, ethyl acetoacetate $(\mathbf{5}, 1 \mathrm{mmol})$ and $10 \mathrm{mg}$ boric acid functionalized MNPs $(\mathbf{1}, 10 \mathrm{mg})$ were added to EtOH 96\% ( $2 \mathrm{~mL})$. The obtained mixture was stirred under reflux condition. After completion of the reaction monitored by TLC (eluent: 25\% v/v EtOAc/n-hexane), EtOH 96\% (3 $\mathrm{mL}$ ) was added and the catalyst 1 was easily separated from the reaction mixture by an external magnet during recrystallization of the products. 


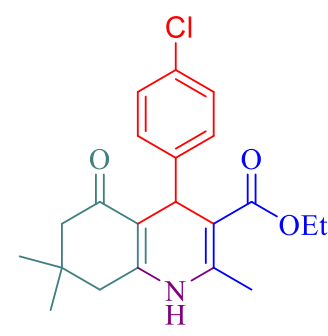

6a

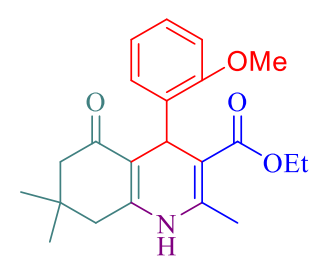

$6 \mathbf{b}$

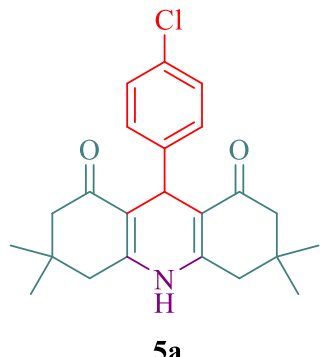

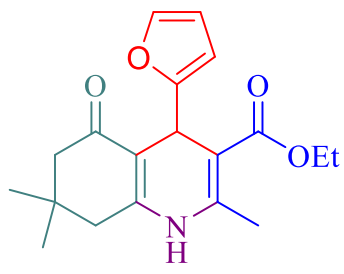

6c

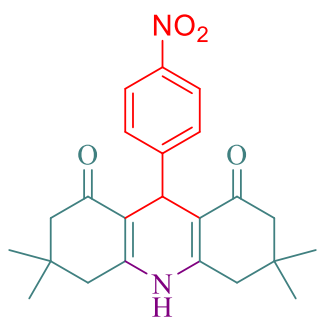

$\mathbf{5 b}$

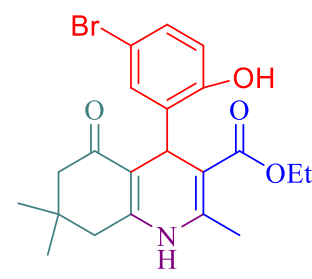

6d

Scheme 2. Scope of Polyhydroquinoline (6a-d) and polyhydroacridines (5a-b) derivatives

\section{Results and discussion}

The catalytic facility of the boric acid functionalized MNPs (1) was evaluated in catalyzing a reaction for the efficient synthesis of Polyhydroquinoline and polyhydroacridines derivatives by condensing indole, various aldehydes, dimedone, ethyl acetoacetate and $\mathrm{NH}_{4} \mathrm{OAc}$ in $\mathrm{EtOH}$ under reflux condition. The results were evaluated qualitatively through TLC. It was found that the quantitative yield can be achieved when the reaction was carried out in the presence of $0.01 \mathrm{~g}$ catalyst. The results are summarized in scheme 2 . The boric acid functionalized MNPs (1) were easily separated via an external magnet and there covered catalyst was reused for at least 6 runs without significant degradation in catalytic activity and performance.

\section{Conclusion}

In conclusion, the new magnetic nano catalyst, factionalized with boric acid, was used as a mild, recyclable and recoverable catalyst for the one-pot synthesis of polyhydroquinolines and polyhydroacridines. It efficiently catalyzed the reaction of different aldehydes, dimedone, ammonium acetate, ethyl acetoacetate in EtOH under reflux 
condition to produce polyhydroquinolines and polyhydroacridines derivatives. Furthermore, this mild nano catalyst have been recovered and reused six times without notable loss of properties. Using $\mathrm{EtOH}$ as a green solvent in the reaction is an indicator of it's being environmentally friendly. Besides, low catalyst loading, short reaction times, mild reaction condition, high yields, recoverable and reusable for several times, ease of separation by an external magnetic field and non-toxication are among the other achieved outcomes of this study.

\section{Acknowledgements}

We are grateful for the financial support from The Research Council of Iran University of Science and Technology (IUST), Tehran, Iran (Grant no. 160/8034).

\section{Refrences}

[1] B. Huang, L. Zeng, Y. Shen, S.J.A.C.I.E. Cui, One-Pot Multicomponent Synthesis of $\beta$-Amino Amides, Angew. Chem. Int. Ed., 56 (2017) 4565-4568.

[2] A. Domling, W. Wang, K.J.C.r. Wang, Chemistry and biology of multicomponent reactions, Chemical reviews, 112 (2012) 3083-3135.

[3] P. Martins, J. Jesus, S. Santos, L. Raposo, C. Roma-Rodrigues, P. Baptista, A.J.M. Fernandes, Heterocyclic anticancer compounds: recent advances and the paradigm shift towards the use of nanomedicine's tool box, Molecules, 20 (2015) 16852-16891.

[4] G. Mathew, J. Lincy, J.J.T.P.I. Chippy, Synthesis, characterization and biological screening of novel 1, 4dihydropyridine derivatives for certain pharmacological activities, The Pharma Innovation, 6 (2017) 165.

[5] A.I. Gutiérrez-Bonet, C. Remeur, J.K. Matsui, G.A.J.J.o.t.A.C.S. Molander, Late-Stage C-H Alkylation of Heterocycles and 1, 4-Quinones via Oxidative Homolysis of 1, 4-Dihydropyridines, Journal of the American Chemical Society, 139 (2017) 12251-12258.

[6] S. Karhale, M. Patil, G. Rashinkar, V.J.R.o.C.I. Helavi, Green and cost effective protocol for the synthesis of 1, 8-dioxo-octahydroxanthenes and 1, 8-dioxo-decahydroacridines by using sawdust sulphonic acid, Research on Chemical Intermediates, 43 (2017) 7073-7086.

[7] J.E. Gómez-Galeno, C. Hurtado, J. Cheng, C. Yardimci, M. Mercola, J.R.J.B. Cashman, b-Annulated 1, 4dihydropyridines as Notch inhibitors, Bioorganic medicinal chemistry letters, 28 (2018) 3363-3367.

[8] M. Nasr-Esfahani, D. Elhamifar, T. Amadeh, B.J.R.A. Karimi, Periodic mesoporous organosilica with ionic-liquid framework supported manganese: an efficient and recyclable nanocatalyst for the unsymmetric Hantzsch reaction, RSC Advances, 5 (2015) 13087-13094.

[9] M.J.J.o.A.C. Wainwright, Acridine-a neglected antibacterial chromophore, Journal of Antimicrobial Chemotherapy, 47 (2001) 1-13. 